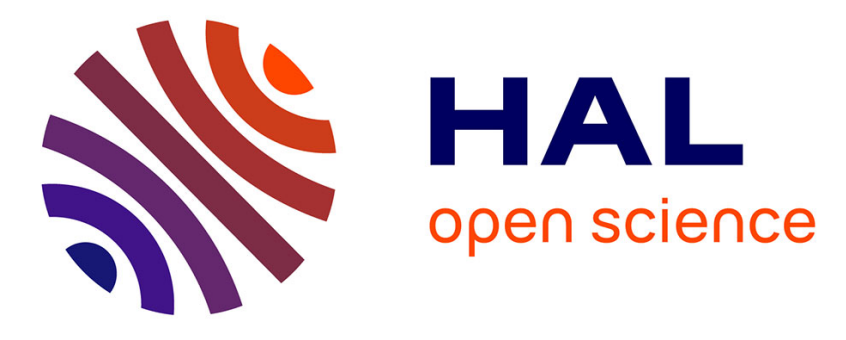

\title{
Proximal method for geometry and texture image decomposition
}

\author{
Luis M. Briceno-Arias, Patrick Louis Combettes, Jean-Christophe Pesquet, \\ Nelly Pustelnik
}

\section{- To cite this version:}

Luis M. Briceno-Arias, Patrick Louis Combettes, Jean-Christophe Pesquet, Nelly Pustelnik. Proximal method for geometry and texture image decomposition. IEEE International Conference on Image Processing (ICIP), Sep 2010, Hong-Kong, Hong Kong SAR China. pp.x+4. hal-00826120

\section{HAL Id: hal-00826120 \\ https://hal.science/hal-00826120}

Submitted on 26 May 2013

HAL is a multi-disciplinary open access archive for the deposit and dissemination of scientific research documents, whether they are published or not. The documents may come from teaching and research institutions in France or abroad, or from public or private research centers.
L'archive ouverte pluridisciplinaire HAL, est destinée au dépôt et à la diffusion de documents scientifiques de niveau recherche, publiés ou non, émanant des établissements d'enseignement et de recherche français ou étrangers, des laboratoires publics ou privés. 


\title{
PROXIMAL METHOD FOR GEOMETRY AND TEXTURE IMAGE DECOMPOSITION
}

\author{
L. M. Briceño-Arias, ${ }^{1}$ P. L. Combettes, ${ }^{1}{ }^{J} .-$ C. Pesquet,${ }^{2}$ and N. Pustelnik ${ }^{2}$ \\ ${ }^{1}$ UPMC Université Paris 06, Laboratoire Jacques-Louis Lions UMR CNRS 7598, 75005 Paris, France \\ ${ }^{2}$ Université Paris-Est, Lab. d'Informatique Gaspard Monge UMR CNRS 8049, 77454 Marne la Vallée Cedex 2, France
}

\begin{abstract}
We propose a variational method for decomposing an image into a geometry and a texture component. Our model involves the sum of two functions promoting separately properties of each component, and of a coupling function modeling the interaction between the components. None of these functions is required to be differentiable, which significantly broadens the range of decompositions achievable through variational approaches. The convergence of the proposed proximal algorithm is guaranteed under suitable assumptions. Numerical examples are provided that show an application of the algorithm to image decomposition and restoration in the presence of Poisson noise.
\end{abstract}

Index Terms - Convex optimization, denoising, image decomposition, image restoration, proximity operator.

\section{INTRODUCTION}

An important problem in image processing is to decompose an image in two elementary structures. In the context of denoising, this decomposition was achieved in [12] with a total variation potential. In [10], a different potential was used to better penalize strongly oscillating components. The resulting variational problem is not straightforward. Numerical methods were proposed in $[3,13]$ and experiments were performed for image denoising and analysis problems based on a geometry-texture decomposition. Another interesting problem is to extract meaningful components from a blurred and noise-corrupted image. In the presence of additive Gaussian noise, a decomposition into geometry and texture components is proposed in [2]. The method developed in the present paper, will make it possible to consider general (not necessarily additive and Gaussian) noise models and arbitrary linear degradation operator. In addition, it lends itself to the incorporation of various additional convex constraints and parallel computing.

In mathematical terms, our problem is to decompose an image $\bar{x} \in \mathbb{R}^{N}$ into the sum of a geometry and a texture component, say

$$
\bar{x}=R_{1}\left(\bar{x}_{1}\right)+R_{2}\left(\bar{x}_{2}\right),
$$

This work was supported by the Agence Nationale de la Recherche under grants ANR-08-BLAN-0294-02 and ANR-09-EMER-004-03. where $R_{1}: \mathbb{R}^{N_{1}} \mapsto \mathbb{R}^{N}$ and $R_{2}: \mathbb{R}^{N_{2}} \mapsto \mathbb{R}^{N}$ are known operators. The vectors $\bar{x}_{1} \in \mathbb{R}^{N_{1}}$ and $\bar{x}_{2} \in \mathbb{R}^{N_{2}}$ to be estimated parameterize, respectively, the geometry and the texture components. They will be obtained via the following variational formulation, which involves potentials $f_{1}$ and $f_{2}$ promoting the properties of $\bar{x}_{1}$ and $\bar{x}_{2}$ separately, as well as a coupling term $\varphi$ modeling their interaction.

Problem 1.1 Let $\left.\left.f_{1}: \mathbb{R}^{N_{1}} \rightarrow\right]-\infty,+\infty\right], f_{2}: \mathbb{R}^{N_{2}} \rightarrow$ ]$-\infty,+\infty]$, and $\left.\left.\varphi: \mathbb{R}^{N_{1}} \times \mathbb{R}^{N_{2}} \rightarrow\right]-\infty,+\infty\right]$ be proper lower semicontinuous convex functions. The problem is to

$$
\underset{x_{1} \in \mathbb{R}^{N_{1}, x_{2}} \in \mathbb{R}^{N_{2}}}{\operatorname{minimize}} f_{1}\left(x_{1}\right)+f_{2}\left(x_{2}\right)+\varphi\left(x_{1}, x_{2}\right) .
$$

Instances of Problem 1.1 have already been studied in $[2,3,4,5,7,9,10,13]$. However, in each case, the coupling function $\varphi$ was differentiable, which excludes many important problems. The objective of the present paper is to remove this restriction and to propose a proximal splitting method for solving (2).

In the next section, we provide some background on proximity operators. In Section 3, we introduce the Parallel ProXimal Algorithm (PPXA), which will be used to solve a decomposed version of Problem 1.1, more amenable to numerical solution. Finally, in Section 4, we describe an application of the proposed framework to image restoration and decomposition in the presence of Poisson noise.

\section{PROXIMITY OPERATORS}

Throughout this paper, we denote by $\mathbb{R}^{K}$ the usual $K$ dimensional Euclidean space and by $\mathrm{I}$ the identity matrix. $\Gamma_{0}\left(\mathbb{R}^{K}\right)$ denotes the class of lower semicontinuous convex functions $\left.\left.f: \mathbb{R}^{K} \rightarrow\right]-\infty,+\infty\right]$ which are proper in the sense that $\operatorname{dom} f=\left\{y \in \mathbb{R}^{K} \mid f(y)<+\infty\right\} \neq \varnothing$. Let $f \in \Gamma_{0}\left(\mathbb{R}^{K}\right)$. For every $y \in \mathbb{R}^{K}$, the function $z \mapsto$ $f(z)+\|y-z\|^{2} / 2$ has a unique minimizer, which is denoted by $\operatorname{prox}_{f} z[11]$. Thus, the proximity operator of $f$ is

$$
\operatorname{prox}_{f}: y \mapsto \underset{z \in \mathbb{R}^{K}}{\operatorname{argmin}} f(z)+\frac{1}{2}\|y-z\|^{2} .
$$

Let $C$ be a nonempty convex subset of $\mathbb{R}^{K}$. Then $\iota_{C}$ denotes the indicator function of $C$ (it takes on the value 0 in $C$ and 
$+\infty$ in $\mathbb{R}^{K} \backslash C$ ), ri $C$ the relative interior of $C$, and, if $C$ is closed, $P_{C}=\operatorname{prox}_{\iota_{C}}$ its projection operator. For a detailed account of the theory of proximity operators, see [9] and the pioneering work in [11]. Closed-form expressions of proximity operators can be found in $[7,8,9,11]$ and the references therein.

The following fact will be used subsequently.

Lemma 2.1 Let $\chi>0$ and set

$$
f: \mathbb{R}^{2} \rightarrow \mathbb{R}:\left(\eta_{1}, \eta_{2}\right) \mapsto \chi \sqrt{\left|\eta_{1}\right|^{2}+\left|\eta_{2}\right|^{2}} .
$$

Then, for every $\left(\eta_{1}, \eta_{2}\right) \in \mathbb{R}^{2}$,

$$
\begin{aligned}
& \operatorname{prox}_{f}\left(\eta_{1}, \eta_{2}\right)= \\
& \begin{cases}\left(1-\frac{\chi}{\sqrt{\left|\eta_{1}\right|^{2}+\left|\eta_{2}\right|^{2}}}\right)\left(\eta_{1}, \eta_{2}\right), & \text { if } \sqrt{\left|\eta_{1}\right|^{2}+\left|\eta_{2}\right|^{2}}>\chi ; \\
(0,0), & \text { otherwise. }\end{cases}
\end{aligned}
$$

\section{DECOMPOSITION: PRODUCT SPACE PPXA}

Problem 1.1 can be rewritten as

$$
\underset{x_{1} \in \mathbb{R}^{N_{1}}, x_{2} \in \mathbb{R}^{N_{2}}}{\operatorname{minimize}} h\left(x_{1}, x_{2}\right)+\varphi\left(x_{1}, x_{2}\right),
$$

where $h:\left(x_{1}, x_{2}\right) \mapsto f_{1}\left(x_{1}\right)+f_{2}\left(x_{2}\right)$. Since $h$ is separable, $\operatorname{prox}_{h}:\left(x_{1}, x_{2}\right) \mapsto\left(\operatorname{prox}_{f_{1}} x_{1}, \operatorname{prox}_{f_{2}} x_{2}\right)$. Hence, if the proximity operators of $f_{1}$ and $f_{2}$ are easily computable, so is $\operatorname{prox}_{h}$. In addition, if $\operatorname{prox}_{\varphi}$ were also easy to implement, then Douglas-Rachford splitting [8] could be used to solve (5). However, in many cases, the proximity operator of the coupling term $\varphi$ will not be explicit. Our strategy is to derive an equivalent decomposed variational formulation by introducing auxiliary variables and functions. This decomposed problem assumes the following form.

Problem 3.1 Let $\left(h_{j}\right)_{1 \leq j \leq p}$ be proper lower semicontinuous convex functions from $\mathbb{R}^{K_{1}} \times \cdots \times \mathbb{R}^{K_{m}}$ to $\left.]-\infty,+\infty\right]$ satisfying $\bigcap_{j=1}^{p}$ ridom $h_{j} \neq \varnothing$. The problem is to

$$
\underset{y_{1} \in \mathbb{R}^{K_{1}}, \ldots, y_{m} \in \mathbb{R}^{K_{m}}}{\operatorname{minimize}} \sum_{j=1}^{p} h_{j}\left(y_{1}, \ldots, y_{m}\right),
$$

under the assumption that a solution exists.

In practice, the objective is to choose functions $\left(h_{j}\right)_{1 \leq j \leq p}$ for which the proximity operators $\left(\operatorname{prox}_{h_{j}}\right)_{1 \leq j \leq p}$ are easily implementable. In turn, this allows us to solve Problem 3.1 by applying [7, Theorem 3.4] in the Euclidean space $\mathbb{R}^{K_{1}} \times$ $\cdots \times \mathbb{R}^{K_{m}}$ as follows.
Theorem 3.1 Let $\left(y_{1, n}\right)_{n \in \mathbb{N}}, \ldots,\left(y_{m, n}\right)_{n \in \mathbb{N}}$ be the sequences generated by the following routine.

Initialization

$$
\mid \begin{aligned}
& \text { Set } \left.\gamma \in] 0,+\infty\left[\text { and take }\left\{\omega_{j}\right\}_{1 \leq j \leq p} \subset\right] 0,1\right] \\
& \text { such that } \sum_{j=1}^{p} \omega_{j}=1 \\
& \text { For } i=1, \ldots, m \\
& \qquad \begin{array}{l}
\text { For } j=1, \ldots, p \\
\left\lfloor\quad s_{i, j, 0} \in \mathbb{R}^{K_{i}}\right. \\
y_{i, 0}=\sum_{j=1}^{p} \omega_{j} s_{i, j, 0}
\end{array}
\end{aligned}
$$

For $n=0,1, \ldots$

$$
\left[\begin{array}{l}
\text { For } j=1, \ldots, p \\
\left\lfloor\quad\left(q_{i, j, n}\right)_{1 \leq i \leq m}=\operatorname{prox}_{\gamma h_{j} / \omega_{j}}\left(s_{i, j, n}\right)_{1 \leq i \leq m}\right. \\
\text { For } i=1, \ldots, m \\
\left\lfloor\begin{array}{l}
y_{i, n+1}=\sum_{j=1}^{m} \omega_{j} q_{i, j, n} \\
\text { For } j=1, \ldots, p \\
\left\lfloor\quad s_{i, j, n+1}=s_{i, j, n}+2 y_{i, n+1}-y_{i, n}-q_{i, j, n} .\right.
\end{array}\right.
\end{array}\right.
$$

Then, for every $i \in\{1, \ldots, m\}$, the sequence $\left(y_{i, n}\right)_{n \in \mathbb{N}}$ converges to a point $y_{i} \in \mathbb{R}^{K_{i}}$, and $\left(y_{1}, \ldots, y_{m}\right)$ is a solution to Problem 3.1.

\section{EXPERIMENTAL RESULTS}

We illustrate the use of the proposed product space PPXA in the context of a simple geometry-texture decomposition from a degraded observation. In our scenario, the observed image $z \in \mathbb{R}^{N}$ of Figure $2(N=512 \times 512)$ is obtained by blurring the original electron microscopy image $\bar{x} \in \mathbb{R}^{N}$ of Figure 1 with a matrix $T \in \mathbb{R}^{N \times N}$, which models a uniform blur of size $5 \times 5$. Furthermore, $\bar{x}$ is contaminated by a Poisson noise with scaling parameter $\alpha=0.6$. We consider a simple instance of (1) with a linear mixture model: $N_{1}=N$, $R_{1}: x_{1} \mapsto x_{1}$, and $R_{2}: x_{2} \mapsto F^{\top} x_{2}$, where $F^{\top} \in \mathbb{R}^{N \times N_{2}}$ is a linear tight frame synthesis operator. In other words, the information regarding the texture component pertains to the coefficients $\bar{x}_{2}$ of its decomposition in the frame. The tightness condition implies that

$$
\left.F^{\top} F=\nu \mathrm{I}, \text { for some } \nu \in\right] 0,+\infty[.
$$

The original image is therefore decomposed as $\bar{x}=\bar{x}_{1}+$ $F^{\top} \bar{x}_{2}$. It is known a priori that $\bar{x} \in C_{1} \cap C_{2}$, where $C_{1}=$ $[0,255]^{N}$ models the constraint on the numerical range of the pixels, and

$$
C_{2}=\left\{\left.x \in \mathbb{R}^{N}\left|\widehat{x}=\left(\eta_{k}\right)_{1 \leq k \leq N}, \sum_{k \in \mathbb{I}}\right| \eta_{k}\right|^{2} \leq \delta\right\}
$$


models an energy bound in the frequency domain $(\widehat{x}$ denotes the 2D Discrete Fourier Transform (DFT) of the image $x$ and II corresponds to some set of discrete frequency indices). In addition, to limit the total variation of the geometrical component, we use the potential $x \mapsto \psi(H x, V x)$, with

$$
\psi:\left(\left(\eta_{k}\right)_{1 \leq k \leq N},\left(\zeta_{k}\right)_{1 \leq k \leq N}\right) \mapsto \chi \sum_{k=1}^{N} \sqrt{\left|\eta_{k}\right|^{2}+\left|\zeta_{k}\right|^{2}},
$$

where $\chi \in] 0,+\infty\left[\right.$, and where $H \in \mathbb{R}^{N \times N}$ and $V \in \mathbb{R}^{N \times N}$ are matrix representations of the horizontal and vertical discrete differentiations, respectively. Furthermore, to promote the sparsity in the frame of the texture component of the image, we introduce the potential

$$
f_{2}:\left(\eta_{k}\right)_{1 \leq k \leq N_{2}} \mapsto \sum_{k=1}^{N_{2}} \tau_{k}\left|\eta_{k}\right|,
$$

where $\left.\left\{\tau_{k}\right\}_{1 \leq k \leq N_{2}} \subset\right] 0,+\infty[$. Finally, as a data fidelity term, we use the generalized Kullback-Leibler divergence $D$, which is well adapted to Poisson noise. Altogether, we arrive at the variational problem

$$
\min _{\substack{x_{1} \in \mathbb{R}^{N}, x_{2} \in \mathbb{R}^{N_{2}} \\ x_{1}+F^{\top} x_{2} \in C_{1} \\ x_{1}+F^{\top} x_{2} \in C_{2}}} \psi\left(H x_{1}, V x_{1}\right)+f_{2}\left(x_{2}\right)+D\left(z, T x_{1}+T F^{\top} x_{2}\right),
$$

which is a particular case of (2) with $f_{1}: x \mapsto \psi(H x, V x)$ and

$$
\begin{aligned}
\varphi:\left(x_{1}, x_{2}\right) \mapsto & D\left(z, T x_{1}+T F^{\top} x_{2}\right)+ \\
& \iota_{C_{1}}\left(x_{1}+F^{\top} x_{2}\right)+\iota_{C_{2}}\left(x_{1}+F^{\top} x_{2}\right) .
\end{aligned}
$$

Since $\operatorname{prox}_{\varphi}$ and $\operatorname{prox}_{f_{1}}$ are not easily computable, a strategy is to decompose (12) into the equivalent problem

$$
\begin{gathered}
\min _{\left(y_{1}, y_{2}, y_{3}, y_{4}, y_{5}, y_{6}\right)} \psi\left(y_{5}, y_{6}\right)+f_{2}\left(y_{2}\right)+D\left(z, y_{4}\right), \\
y_{3}=y_{1}+F^{\top} y_{2} \\
y_{3} \in C_{1}, y_{3} \in C_{2} \\
y_{4}=T y_{3} \\
y_{5}=H y_{1}, y_{6}=V y_{1}
\end{gathered}
$$

where we have changed the variables $\left(x_{1}, x_{2}\right)$ into $\left(y_{1}, y_{2}\right)$ and introduced the auxiliary variables $\left(y_{3}, y_{4}, y_{5}, y_{6}\right)$. Problem (14) is a particular case of (6) with $m=6, p=3$, $K_{1}=K_{3}=K_{4}=K_{5}=K_{6}=N, K_{2}=N_{2}$, and

$$
\left\{\begin{aligned}
h_{1}:\left(y_{1}, \ldots, y_{6}\right) & \mapsto f_{2}\left(y_{2}\right)+\iota_{C_{1}}\left(y_{3}\right)+D\left(z, y_{4}\right) \\
& +\psi\left(y_{5}, y_{6}\right), \\
h_{2}:\left(y_{1}, \ldots, y_{6}\right) & \mapsto \iota_{C_{2}}\left(y_{3}\right), \\
h_{3}:\left(y_{1}, \ldots, y_{6}\right) & \mapsto \iota_{\{0\}}\left(y_{1}+F^{\top} y_{2}-y_{3}\right) \\
& +\iota_{\{0\}}\left(T y_{3}-y_{4}\right)+\iota_{\{0\}}\left(H y_{1}-y_{5}\right) \\
& +\iota_{\{0\}}\left(V y_{1}-y_{6}\right) .
\end{aligned}\right.
$$

In [1], a similar reformulation is considered in the case when $m=2$, and solved by an alternating direction method of multipliers.

The proximity operators associated with $f_{2}$ and $D(z, \cdot)$ can be obtained from [9]. On the other hand, $\operatorname{prox}_{\psi}$ is derived from Lemma 2.1 and, as seen earlier, $\operatorname{prox}_{\iota_{C_{1}}}=P_{C_{1}}$ and $\operatorname{prox}_{\iota_{C_{2}}}=P_{C_{2}}$. Furthermore, if we set

$$
\boldsymbol{L}=\left[\begin{array}{cccccc}
\mathrm{I} & F^{\top} & -\mathrm{I} & 0 & 0 & 0 \\
0 & 0 & T & -\mathrm{I} & 0 & 0 \\
H & 0 & 0 & 0 & -\mathrm{I} & 0 \\
V & 0 & 0 & 0 & 0 & -\mathrm{I}
\end{array}\right]
$$

we deduce from (15) that $h_{3}=\iota_{\{0\}} \circ \boldsymbol{L}$. Lastly, since the matrices $T, H$, and $V$ are associated with periodic convolution operators, they are diagonalized by the DFT. Hence, using (8), $\operatorname{prox}_{h_{3}}$ can be deduced from the well-known expression of the projection onto the kernel of $\boldsymbol{L}$.

The convergence of the employed algorithm is guaranteed under the assumptions of Problem 3.1. Since int $\left(C_{1} \cap C_{2}\right) \neq$ $\varnothing$, these assumptions are satisfied due to the fact that $T$ models a uniform blur and thus has positive entries and each of its lines is nonzero.

Figure 3 shows the results of the decomposition into geometry and texture components. The parameter $\chi$ of (10) and the parameters $\left(\tau_{k}\right)_{1 \leq k \leq N_{2}}$ of (11) are selected so as to maximize the signal-to-noise ratio (SNR). The matrix $F$ is a tight frame version of the dual-tree transform proposed in [6] using symlets of length 6 over 3 resolution levels $(\nu=2$ and $\left.N_{2}=2 N\right)$. The same discrete gradient matrices $H$ and $V$ as in [7, Section 4.2] are used.

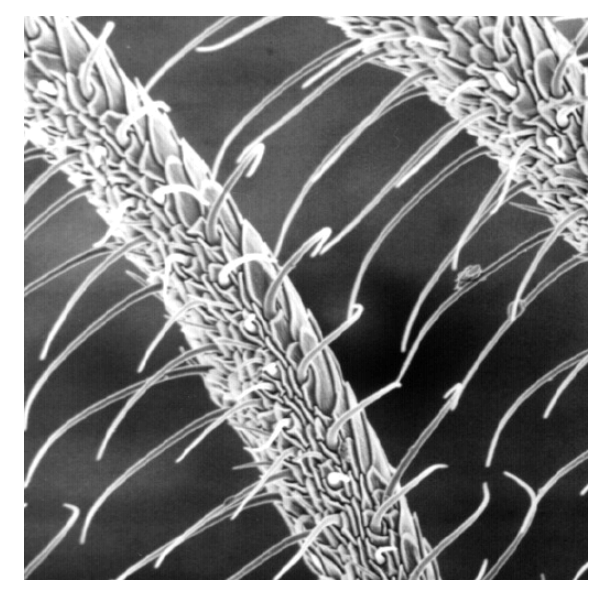

Fig. 1. Original image $\bar{x}$.

\section{REFERENCES}

[1] M. V. Afonso, J. M. Bioucas-Dias, M. A. T. Figueiredo "Fast image recovery using variable splitting and constrained optimization," http://arxiv.org/abs/0910.4887.

[2] S. Anthoine, E. Pierpaoli, and I. Daubechies, "Deux méthodes de déconvolution et séparation simultanées - Application à la 


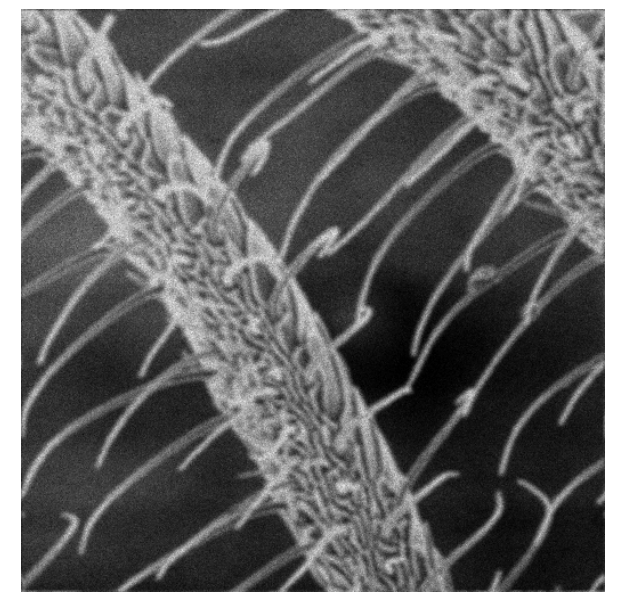

Fig. 2. Degraded image $z: \operatorname{SNR}=15.7 \mathrm{~dB}-\operatorname{SSIM}=0.55$.

reconstruction des amas de galaxies," Traitement Signal, vol. 23, pp. 439-447, 2006.

[3] J.-F. Aujol, G. Aubert, L. Blanc-Féraud, and A. Chambolle, "Image decomposition into a bounded variation component and an oscillating component," J. Math. Imaging Vision, vol. 22, pp. 71-88, 2005.

[4] J.-F. Aujol, G. Gilboa, T. Chan, and S. Osher, "Structuretexture image decomposition - Modeling, algorithms, and parameter selection," Int. J. Comput. Vision, vol. 67, pp. 111136, 2006

[5] L. M. Briceño-Arias and P. L. Combettes, "Convex variational formulation with smooth coupling for multicomponent signal decomposition and recovery," Numer. Math. Theory Methods Appl., vol. 2, pp. 485-508, 2009.

[6] C. Chaux, L. Duval, and J.-C. Pesquet, "Image analysis using a dual-tree $M$-band wavelet transform," IEEE Trans. Image Process., vol. 15, pp. 2397-2412, 2006.

[7] P. L. Combettes and J.-C. Pesquet, "A proximal decomposition method for solving convex variational inverse problems," Inverse Problems, vol. 24, Article ID 065014, 27 pp., 2008.

[8] P. L. Combettes and J.-C. Pesquet, "Proximal splitting methods in signal processing," http://arxiv.org/abs/0912.3522.

[9] P. L. Combettes and V. R. Wajs, "Signal recovery by proximal forward-backward splitting," Multiscale Model. Simul., vol. 4, pp. 1168-1200, 2005.

[10] Y. Meyer, "Oscillating patterns in image processing and in some nonlinear evolution equations," AMS, Providence, RI, 2001.

[11] J.-J. Moreau, "Proximité et dualité dans un espace Hilbertien," Bull. Soc. Math. France, vol. 93, pp. 273-299, 1965.

[12] L. I. Rudin, S. Osher, and E. Fatemi, "Nonlinear total variation based noise removal algorithms," Phys. D, vol. 60, pp. 259 $268,1992$.

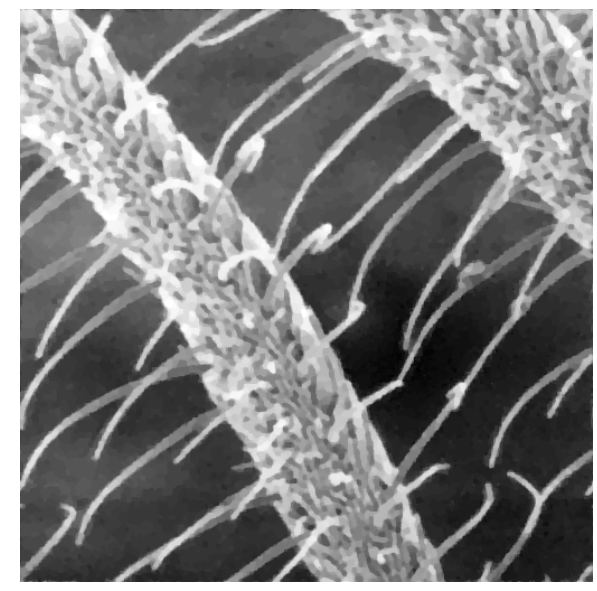

Geometry component $x_{1}$.

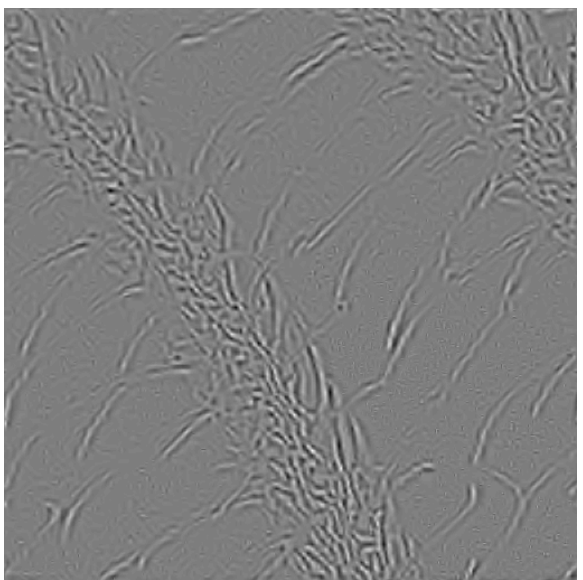

Texture component $F^{\top} x_{2}$.

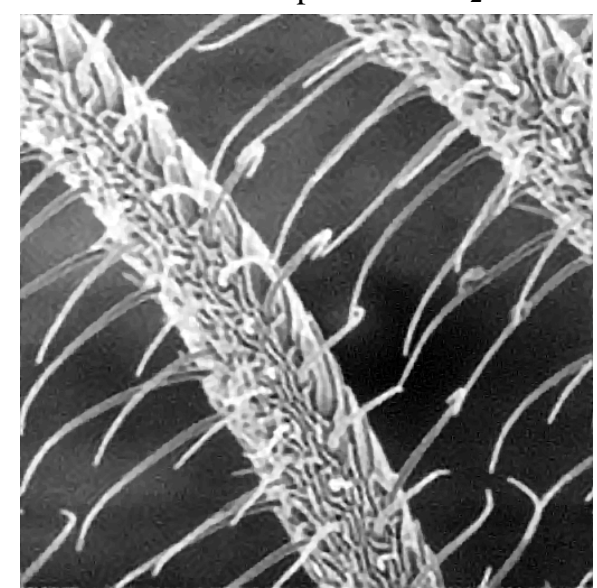

Restoration with PPXA: $x=x_{1}+F^{\top} x_{2}$. $\mathrm{SNR}=19.3 \mathrm{~dB}-\mathrm{SSIM}=0.79$.

Fig. 3. Decomposition and reconstruction results

[13] A. L. Vese and S. Osher, "Modeling textures with total variation minimization and oscillating patterns in image processing," J. Sci. Comput., vol. 15, pp. 553-572, 2003. 